\title{
ANÁLISE RADIOGRÁFICA COMPARATIVA DO TRUNK SHIFT DA ESCOLIOSE IDIOPÁTICA DO ADOLESCENTE ENTRE GANCHOS, HÍBRIDA E PARAFUSOS
}

\author{
COMPARATIVE RADIOGRAPHIC ANALYSIS OF TRUNK SHIFT OF ADOLESCENT IDIOPATHIC \\ SCOLIOSIS BETWEEN HOOKS, SCREWS AND HYBRID
}

\author{
ANÁLISIS COMPARATIVO RADIOLÓGICO DEL TRUNK SHIFT DE LA ESCOLIOSIS IDIOPÁTICA \\ DEL ADOLESCENTE ENTRE LOS GANCHOS, TORNILLOS E HIBRIDAS
}

Cesar Daniel Macedo ${ }^{1}$, Luciano Pellegrino², Ricardo Shigueaki Galhego Umeta ${ }^{3}$, Maria Fernanda Silber Cafaro ${ }^{4}$, Robert Meves ${ }^{5}$,

Elcio Landim ${ }^{6}$, Osmar AvanzI ${ }^{7}$

RESUMO

Objetivos: Analisar radiograficamente a descompensação lateral do tronco (trunk shift) em pacientes com escoliose idiopática do adolescente submetidos à artrodese seletiva da curva torácica principal nas três diferentes técnicas de gancho, híbridas e parafusos. Métodos: Avaliadas e comparadas retrospectivamente radiografias ântero-posteriores no período pré-operatório, pós-operatório imediato e no seguimento mínimo de um ano em nove pacientes com ganchos, 13 híbridas e 13 parafusos, quanto à mensuração do trunk shift e ângulo de Cobb entre essas três diferentes técnicas de instrumentação. Resultados: As médias no período pré-operatório imediato da descompensação do tronco para direita foram de 16,7 mm para ganchos, 13,2 mm para híbridas, 27,3 mm para parafusos. No pós-operatório imediato foram de $-16 \mathrm{~mm}$ para ganchos, $-12,8 \mathrm{~mm}$ para híbridas, $-10,7 \mathrm{~mm}$ para parafusos, com uma correção no seguimento de um ano para -9,6 mm para ganchos, -11,8 mm para híbridas e -10,7 mm para parafusos ocorrendo o deslocamento do tronco para esquerda. Todos os casos com uso de somente parafusos pediculares apresentaram trunk shift menor que $20 \mathrm{~mm}$. Verificamos desequilíbrio no grupo de ganchos em um paciente $(11,11 \%)$ e de híbridas em dois pacientes $(15,38 \%)$. Conclusão: Não houve diferença significativa na descompensação lateral do tronco entre o uso das três técnicas de instrumentação.

Descritores: Escoliose/cirurgia; Fusão vertebral; Adolescente.

\begin{abstract}
Objectives: To analyze radiographic lateral decompensation of the trunk (trunk shift) in patients with adolescent idiopathic scoliosis who underwent selective fusion of primary thoracic curve in the three different techniques of hook, hybrid and screws. Methods: Evaluation of anteroposterior radiographs retrospectively comparing the preoperative period, immediate postoperative and follow-up of at least one year in nine patients with hooks, 13 hybrid and 13 screws, and the measurement of trunk shift and Cobb angle between these three different instrumentation techniques. Results: The averages in the preoperative period to the right lateral decompensation of the trunk was $16,7 \mathrm{~mm}$ for hooks, 13,2 $\mathrm{mm}$ for hybrids, $27,3 \mathrm{~mm}$ for screws. In the immediate postoperative period were $-16 \mathrm{~mm}$ for hooks, $-12,8 \mathrm{~mm}$ for hybrids, $-10,7 \mathrm{~mm}$ for screws, with a correction in the one year follow-up of $-9.6 \mathrm{~mm}$ for hooks, $-11,8$ for hybrids and $-10,7 \mathrm{~mm}$ for screws occurring displacement of the trunk to the left. All cases using only pedicle screws showed trunk shift of less than $20 \mathrm{~mm}$. We observed an imbalance in the group of hooks in one patient (11.11\%) and of hybrids in two patients (15.38\%). Conclusion: No significant differences in trunk lateral decompensation between the use of the three instrumentation techniques.
\end{abstract}

Keywords: Scoliosis/surgery; Spinal fusion; Adolescent.

\section{RESUMEN}

Objetivos: Analizar, radiográficamente, la descompensación del tronco (trunk shift: desplazamiento del tronco) en pacientes con escoliosis idiopática del adolescente sometidas a artrodesis selectivas de la curva torácica primaria en las tres diferentes técnicas: ganchos, hibridas y tornillos. Métodos: Evaluación y comparación retrospectiva de las radiografías anteroposteriores en los períodos preoperatorio, posoperatorio y d el seguimiento de, al menos. 1 año en 9 pacientes con ganchos, 13 híbridas y 13 con tornillos, cuanto a la medición del desplazamiento del tronco y del ángulo de Cobb entre estas tres diferentes técnicas de instrumentación. Resultados: Los promedios en el periodo preoperatorio inmediato referentes a la descompensación del tronco hacia la derecha fueron 16,7 mm ganchos, híbridas 13,2 mm, 27,3 mm de tornillos. En el posoperatorio inmediato, fueron -16 mm ganchos, híbridas -12,8 mm, -10,7 mm tornillos, con una corrección, después de un año de seguimiento, a -9,6 $\mathrm{mm}$ para los ganchos, $-11,8 \mathrm{~mm}$ para híbridas y $-10,7 \mathrm{~mm}$ para tornillos, ocurriendo el desplazamiento

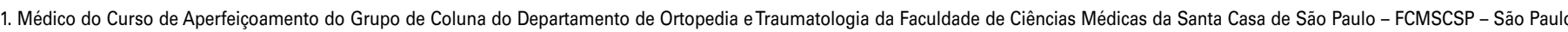
(SP), Brasil.

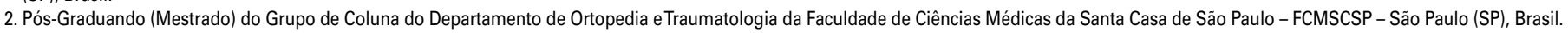

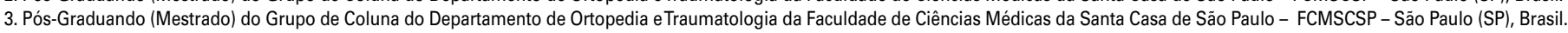

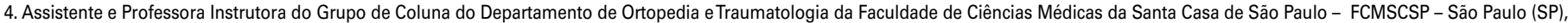
Brasil.

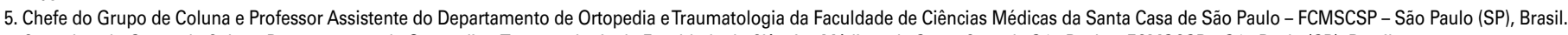
6. Consultor do Grupo de Coluna Departamento de Ortopedia eTraumatologia da Faculdade de Ciências Médicas da Santa Casa de São Paulo - FCMSCSP - São Paulo (SP), Brasil.

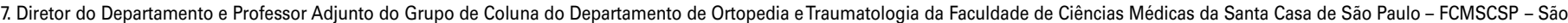
Paulo (SP), Brasil.
} 
del tronco hacia la izquierda. Todos los casos con solamente el uso de tornillos de pedículo mostraron desplazamiento del tronco menor que $20 \mathrm{~mm}$. Se observó un desequilibrio en el grupo de ganchos en una paciente $(11,11 \%)$ y en la hibridación en dos pacientes (15,38\%). Conclusión: No hubo diferencia significativa en el desplazamiento lateral del tronco en el uso de las tres técnicas de instrumentación.

Descriptores: Escoliosis/cirugía; Fusión vertebral; Adolescente.

\section{INTRODUÇÃO}

O avanço dos novos tipos de instrumentação na escoliose idiopática do adolescente, possibilitaram as maiores correções das deformidades. Nas artrodeses seletivas, as quais realizadas somente instrumentação da curva torácica principal, as curvas classificadas por King como tipo II e Lenke 2CN ${ }^{1,2}$ (componente torácico é maior e menos flexível que o componente lombar) obtiveram um menor numero de vértebras na artrodese, possibilitando uma maior mobilidade, menor tempo e menor risco cirúrgicos ${ }^{1,3}$

O objetivo na correção da curva foi alcançado com o uso do instrumental de $3^{\mathrm{a}}$ geração, porém foram observados em diversos estudos, após seguimento de 1 ano, a presença da descompensação do tronco ${ }^{3-6}$. Vários métodos foram descritos na literatura para avaliar a descompensação pós-operatória visando estudar esta complicação.

A descompensação, é possível complicação que ocorre após a instrumentação seletiva das curvas de King tipo 2 e Lenke $2 \mathrm{CN}^{1,2}$. A Scoliosis Research Society (SRS) define como "compensação" quando há alinhamento vertical do ponto médio de C7 com o ponto médio do sacro no plano coronal. Esta definição certamente descreve a posição da cabeça sobre a pelve. A descompensação ocorre quando há o desalinhamento da linha média superior a 2 centímetros ${ }^{4}$.

Segundo Richards et al. ${ }^{6}$, um paciente com escoliose torácica pode ter equilíbrio perfeito entre a cabeça sobre a pelve e ser descrito como tendo nenhuma descompensação (posição do cabeça sobre a pelve). No entanto, se este mesmo indivíduo tem uma grande curva torácica, o tronco pode ser deslocado significativamente longe da linha média da pelve, refletindo uma notável descompensação (posição do tórax sobre a pelve) ${ }^{4,6}$. A verdadeira medida da descompensação do tronco, conforme este autor, é feita com o trunk shit (deslocamento lateral do tronco, Figura 1) e não com a técnica preconizada da SRS ${ }^{7}$.

Neste estudo realizaremos a análise radiográfica e comparativa das possíveis técnicas de instrumentação seletivas, através da fixação por ganchos, hibrida e parafusos pediculares quanto à descompensação lateral do tronco (trunk shift).

\section{MATERIAIS E MÉTODOS}

A avaliação deste estudo retrospectivo, aprovado pelo Cep $n^{\circ}$ 1867, foi estabelecida conforme a classificação de King tipo II e Lenke $2 \mathrm{CN}^{1,2}$ em pacientes com escoliose idiopática do adolescente. Foram selecionados os pacientes com radiografias de inclinação apresentando flexibilidade da curva lombar, possibilitando uma artrodese seletiva da curva torácica rígida 4 .

No período do ano de 1993 a 2010 foram avaliadas as radiografias antero-posteriores de 35 pacientes King tipo II e Lenke $2 \mathrm{CN}^{1,2}$ com a mensuração do trunk shift e ângulo de Cobb nas três diferentes técnicas de instrumentação seletiva: fixação por ganchos, híbrida e parafusos pediculares.

As radiografias foram fornecidas dos arquivos do hospital Santa Casa de São Paulo (SAME-ISCMSP). Os critérios de inclusão foram prontuários que continham radiografias antero-posteriores, com a presença dos exames pré operatório, pós-operatórios imediato e após segmento de no mínimo 1 ano da cirurgia. Foram escolhidos pacientes submetidos à fusão seletiva (da vértebra L1 ou proximalmente a mesma) ${ }^{8}$. Foram excluídos pacientes com outros tipos de curva e artrodeses realizadas até a vértebra de L2 ou abaixo.

A medida do trunk shift (método conhecido como desloca- mento lateral do tronco) é a distância horizontal entre o centro da pelve ao ponto médio que corta a linha traçada entre as extremidades das $\operatorname{costelas}^{6}$ (Figura 1). Esta medida foi realizada e analisada juntamente com o ângulo de Cobb nas radiografias antero-posteriores, observando sua evolução durante os períodos pré operatório, pós-operatório imediato e segmento tardio.

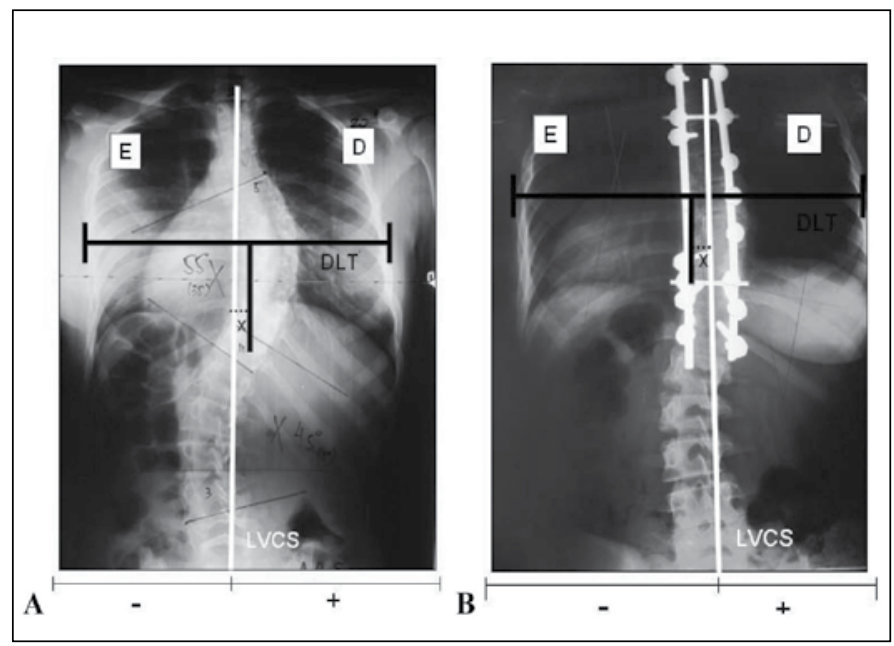

Figura 1. As radiografia de um paciente com escoliose idiopática do adolescente e a analise do trunk shift pré e pós-operatória com uso de parafusos pediculares. A) radiografia pré operatória mostrando o deslocamento lateral do tronco (DLT), distancia " $X$ " para direita da linha vertical central do sacro (LVCS) B) radiografia do pós-operatório imediato demonstrando o deslocamento do tronco para esquerda.

\section{ANÁLISE ESTATÍSTICA}

Para comparação dos resultados dos três tipos de instrumentação, avaliando-se a hipótese de igualdade entre eles, foi considerado o teste não paramétrico de Kruskal-Wallis. No caso da rejeição da hipótese de igualdade dos três tipos de instrumentação, estas foram comparadas duas a duas. Valores de $p$ menores do que 0,05 indicaram significância estatística.

\section{RESULTADOS}

A média de idade é de 14 anos e 11 meses. Foram analisados as técnicas de instrumentação de $3^{\circ}$ geração no total de 35 pacientes sendo 9 pacientes com gancho, 13 pacientes com híbrida e 13 com parafusos. Os níveis de instrumentação variaram de T3 a L1. (Quadro 1 a 3)

A média dos ângulos de Cobb nas radiografias foram obtidas conforme a Tabela 1.

\section{Comparação das médias do trunk shift entre os períodos pré- operatório e seguimento de 1 ano}

As médias no período pré operatório imediato da descompensação do tronco foram de $16,7 \mathrm{~mm}$ ganchos, $13,2 \mathrm{~mm}$ híbridas, $27,3 \mathrm{~mm}$ parafusos, com uma correção no seguimento de 1 ano para $-9,6 \mathrm{~mm}$ para ganchos, $-11,8 \mathrm{~mm}$ híbrida e $-10,7 \mathrm{~mm}$ para parafusos. Em todos os grupos a descompensação do tronco foi corrigida da direita valores positivos no Quadro 1. 1) para esquerda (valores negativos no Quadro 2 e 3 ), porém não houve uma diferença significativa da correção do trunk shift entre os três grupos (valor do $\mathrm{p}=0,058$ ) (Tabela 1 ). 
Quadro 1. Resultados obtidos com instrumentação com Ganchos

\begin{tabular}{|c|c|c|c|c|c|c|c|c|c|}
\hline Nivel & $\begin{array}{c}\text { Ângulo da Curva } \\
\text { Torácica Inicial }\end{array}$ & $\begin{array}{c}\text { Ângulo da } \\
\text { Curva Lombar } \\
\text { Inicial }\end{array}$ & $\begin{array}{l}\text { Trunk Shift } \\
\text { Inicial* }\end{array}$ & $\begin{array}{c}\text { Ângulo da Curva } \\
\text { Torácica } \\
\text { pós-operatório }\end{array}$ & $\begin{array}{c}\text { Ângulo da Curva } \\
\text { Lombar } \\
\text { pós-operatório }\end{array}$ & $\begin{array}{c}\text { Trunk Shift } \\
\text { pós-operatório* }\end{array}$ & $\begin{array}{l}\text { Ângulo da } \\
\text { Curva Torácica } \\
\text { Seguimento }\end{array}$ & $\begin{array}{c}\text { Ângulo da } \\
\text { Curva Lombar } \\
\text { Seguimento }\end{array}$ & $\begin{array}{l}\text { Trunk Shift } \\
\text { Segmento* }\end{array}$ \\
\hline T4-L1 & 60 & 40 & 1,1 & 25 & 22 & $-1,6$ & 32 & 12 & $-1,6$ \\
\hline T5-L1 & 48 & 30 & 0,4 & 20 & 11 & $-0,2$ & 20 & 9 & -1 \\
\hline T4-T12 & 53 & 32 & 3,3 & 26 & 23 & -3 & 30 & 20 & $-0,4$ \\
\hline T5-L1 & 55 & 30 & 2,4 & 32 & 1 & $-2,8$ & 22 & 6 & 0,9 \\
\hline T4-L1 & 49 & 30 & 3 & 12 & 6 & $-1,3$ & 12 & 8 & $-1,5$ \\
\hline T4-T12 & 46 & 42 & 3,7 & 18 & 22 & $-0,7$ & 40 & 32 & $-0,6$ \\
\hline T4-L1 & 68 & 39 & $-0,4$ & 20 & 12 & $-3,5$ & 21 & 22 & $-2,1$ \\
\hline T4-L1 & 55 & 43 & 1 & 29 & 27 & 0 & 20 & 29 & $-1,1$ \\
\hline T5-Т12 & 52 & 43 & 0,5 & 28 & 27 & $-1,3$ & 43 & 31 & $-1,2$ \\
\hline
\end{tabular}

Fonte: Serviço de Arquivo Médico da ISCMSP - SAME.

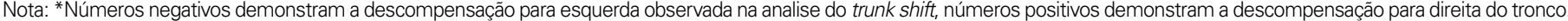

Quadro 2. Resultados obtidos com instrumentação híbrida.

\begin{tabular}{|c|c|c|c|c|c|c|c|c|c|}
\hline Nivel & $\begin{array}{c}\text { Ângulo da } \\
\text { Curva Torácica } \\
\text { Inicial }\end{array}$ & $\begin{array}{l}\text { Ângulo da } \\
\text { Curva Lombar } \\
\text { Inicial }\end{array}$ & $\begin{array}{l}\text { Trunk Shift } \\
\text { Inicial }\end{array}$ & $\begin{array}{l}\text { Ângulo da Curva } \\
\text { Torácica } \\
\text { pós-operatório }\end{array}$ & $\begin{array}{c}\text { Ângulo da Curva } \\
\text { Lombar } \\
\text { pós-operatório }\end{array}$ & $\begin{array}{c}\text { Trunk Shift } \\
\text { pós-operatório }\end{array}$ & $\begin{array}{c}\text { Ângulo da Curva } \\
\text { Torácica } \\
\text { Seguimento }\end{array}$ & $\begin{array}{c}\text { Ângulo da Curva } \\
\text { Lombar Seguimento }\end{array}$ & $\begin{array}{l}\text { Trunk Shift } \\
\text { Segmento }\end{array}$ \\
\hline T4-T12 & 54 & 54 & 2,5 & 30 & 30 & $-2,8$ & 40 & 28 & $-2,4$ \\
\hline Т3-Т12 & 55 & 49 & 0,5 & 50 & 47 & $-2,3$ & 50 & 48 & $-1,6$ \\
\hline T4-T12 & 55 & 45 & 0 & 20 & 10 & 0,3 & 28 & 26 & $-0,1$ \\
\hline T3-L1 & 100 & 60 & 1,6 & 46 & 20 & $-0,8$ & 56 & 22 & $-0,9$ \\
\hline Т3-Т12 & 61 & 35 & 1,1 & 24 & 15 & $-0,2$ & 27 & 20 & $-0,1$ \\
\hline T2-L1 & 56 & 18 & 2,1 & 10 & 5 & $-2,1$ & 17 & 13 & $-1,5$ \\
\hline T4-L1 & 60 & 34 & $-0,9$ & 40 & 30 & $-3,6$ & 32 & 29 & $-2,8$ \\
\hline T3-L1 & 68 & 50 & 1,1 & 35 & 20 & $-1,7$ & 40 & 33 & $-0,9$ \\
\hline T4-L1 & 55 & 32 & 1,4 & 25 & 11 & $-1,2$ & 22 & 22 & $-0,9$ \\
\hline T4-L1 & 70 & 48 & 2,4 & 25 & 17 & 0,6 & 25 & 30 & $-0,6$ \\
\hline T4-T12 & 50 & 50 & 0,5 & 30 & 22 & $-2,2$ & 40 & 32 & $-0,6$ \\
\hline Т3-Т12 & 58 & 39 & 3,2 & 24 & 8 & $-0,6$ & 21 & 26 & $-1,8$ \\
\hline T3-L1 & 70 & 40 & 1,7 & 35 & 12 & $-0,1$ & 20 & 29 & $-1,1$ \\
\hline
\end{tabular}

Fonte: Serviço de Arquivo Médico da ISCMSP - SAME.

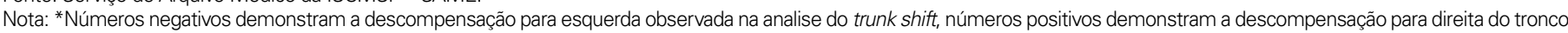

Quadro 3. Resultados obtidos com instrumentação de parafusos pediculares.

\begin{tabular}{|c|c|c|c|c|c|c|c|c|c|}
\hline Nivel & $\begin{array}{c}\text { Ângulo } \\
\text { da Curva } \\
\text { Torácica } \\
\text { Inicial }\end{array}$ & $\begin{array}{c}\text { Ângulo } \\
\text { da Curva } \\
\text { Lombar } \\
\text { Inicial }\end{array}$ & $\begin{array}{c}\text { Trunk } \\
\text { Shift } \\
\text { Inicial }\end{array}$ & $\begin{array}{c}\text { Ângulo da } \\
\text { Curva Torácica } \\
\text { pós-operatório }\end{array}$ & $\begin{array}{c}\text { Ângulo da } \\
\text { Curva Lombar } \\
\text { pós-operatório }\end{array}$ & $\begin{array}{c}\text { Trunk Shift } \\
\text { pós-operatório* }\end{array}$ & $\begin{array}{c}\text { Ângulo da Curva } \\
\text { Torácica } \\
\text { Seguimento }\end{array}$ & $\begin{array}{c}\text { Ângulo da Curva } \\
\text { Lombar } \\
\text { Seguimento }\end{array}$ & $\begin{array}{l}\text { Trunk Shift } \\
\text { Segmento* }\end{array}$ \\
\hline T4-L1 & 60 & 40 & 3,3 & 20 & 8 & $-0,7$ & 25 & 20 & $-0,5$ \\
\hline T5-Т12 & 53 & 30 & 0,3 & 19 & 8 & $-0,7$ & 16 & 12 & -1 \\
\hline T4-L1 & 78 & 50 & 3,8 & 31 & 12 & $-0,6$ & 40 & 32 & -1 \\
\hline T4-L1 & 62 & 32 & 2,6 & 20 & 10 & $-1,3$ & 29 & 35 & $-0,9$ \\
\hline T4-L1 & 75 & 55 & 3,5 & 35 & 25 & $-3,5$ & 40 & 36 & $-1,5$ \\
\hline T3-L1 & 70 & 45 & 4,5 & 17 & 17 & $-0,3$ & 21 & 22 & $-1,8$ \\
\hline T4-T12 & 60 & 45 & 4 & 17 & 12 & $-0,9$ & 21 & 25 & $-0,2$ \\
\hline T4-L1 & 77 & 54 & 3,3 & 45 & 18 & $-0,3$ & 29 & 11 & $-1,2$ \\
\hline T4-L1 & 53 & 51 & 2,5 & 13 & 12 & $-1,2$ & 16 & 13 & $-0,7$ \\
\hline T4-T12 & 62 & 40 & 1,2 & 19 & 21 & $-2,1$ & 33 & 28 & $-1,2$ \\
\hline T4-L1 & 65 & 45 & 2,3 & 23 & 22 & $-1,5$ & 32 & 24 & $-0,9$ \\
\hline T3-L1 & 69 & 55 & 2,4 & 28 & 10 & $-1,9$ & 30 & 12 & $-1,3$ \\
\hline T4-L1 & 49 & 36 & 1,8 & 10 & 5 & $-1,8$ & 11 & 5 & $-1,7$ \\
\hline
\end{tabular}

Fonte: Serviço de Arquivo Médico da ISCMSP - SAME.

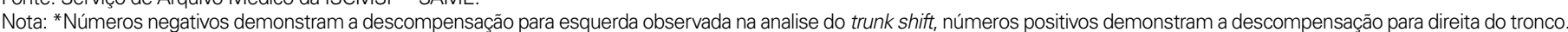


Tabela 1. Médias das curvas torácicas e lombares entre os períodos pré-operatório, pós-operatório e seguimento de um ano entre os três técnicas analisadas.

\begin{tabular}{l|c|c|c|c}
\hline Mensurações das curvas & Gancho & Híbrida & Parafuso & \\
\hline Curva torácica (Cobb) & 54 & 62,46 & 64,08 & $\mathrm{p}=0,024$ \\
\hline ângulo torácico inicial & 23,33 & 30,31 & 22,85 & $\mathrm{p}=0,507$ \\
\hline ângulo torácico pós-operatório & 26,67 & 32,15 & 26,38 & $\mathrm{p}=0,507$ \\
\hline ângulo torácico no seguimento & \multicolumn{5}{|l}{} \\
\hline Curva lombar (Cobb) & 36,56 & 42,62 & 44,46 & $\mathrm{p}=0,070$ \\
\hline ângulo lombar inicial & 16,78 & 19 & 21,15 & $\mathrm{p}=0,478$ \\
\hline ângulo lombar pós-operatório & 18,78 & 27,54 & 21,15 & $\mathrm{p}=0,138$ \\
\hline ângulo lombar no seguimento &
\end{tabular}

Fonte: Serviço de Arquivo Médico da ISCMSP - SAME.

Comparação das médias do trunk shift entre os períodos pósoperatório e seguimento de 1 ano

As médias no período pós-operatório imediato da descompensação do tronco foram de $-16 \mathrm{~mm}$ ganchos, $-12,8 \mathrm{~mm}$ híbridas, $-10,7 \mathrm{~mm}$ parafusos, com uma correçẫo no seguimento de 1 ano para $-9,6 \mathrm{~mm}$ para ganchos, $-11,8 \mathrm{~mm}$ híbrida e $-10,7 \mathrm{~mm}$ para parafusos. Houve pouca diferença das médias entre os três tipos de instrumentação, observado a correção da descompensação lateral do tronco do pós-operatório imediato para o seguimento de 1 ano (Figura 2, valor do $\mathrm{p}=0,949$ ).

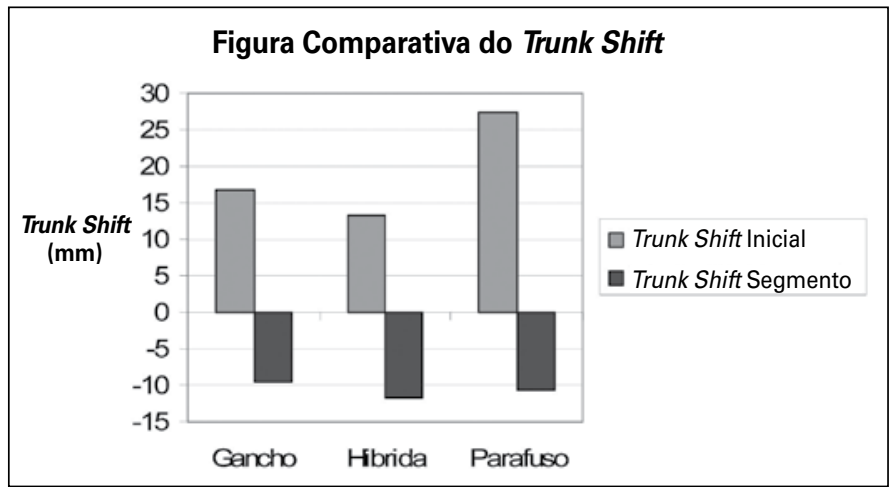

Fonte: Serviço de Arquivo Médico da ISCMSP - SAME.

Figura 2. Médias comparativas entre o trunk shift nas radiografias préoperatório inicial e seguimento de 1 ano.

\section{Descompensação Lateral do Tronco no pós-operatório imediato}

A descompensação lateral do tronco para esquerda, maior e igual a $10 \mathrm{~mm}$ foi maior para o grupo parafusos (Figura 3).

A descompensação lateral do tronco para esquerda, maior e igual a 20mm foi maior para o grupo híbrido e ganchos (Figura 4).

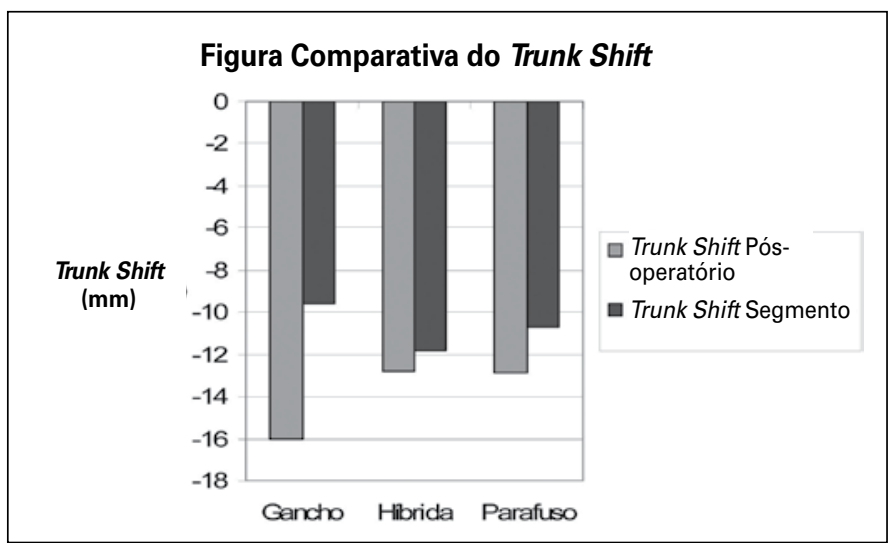

Fonte: Serviço de Arquivo Médico da ISCMSP - SAME.

Figura 3. Médias comparativas entre o trunk shift nas radiografias préoperatório inicial e seguimento de 1 ano.

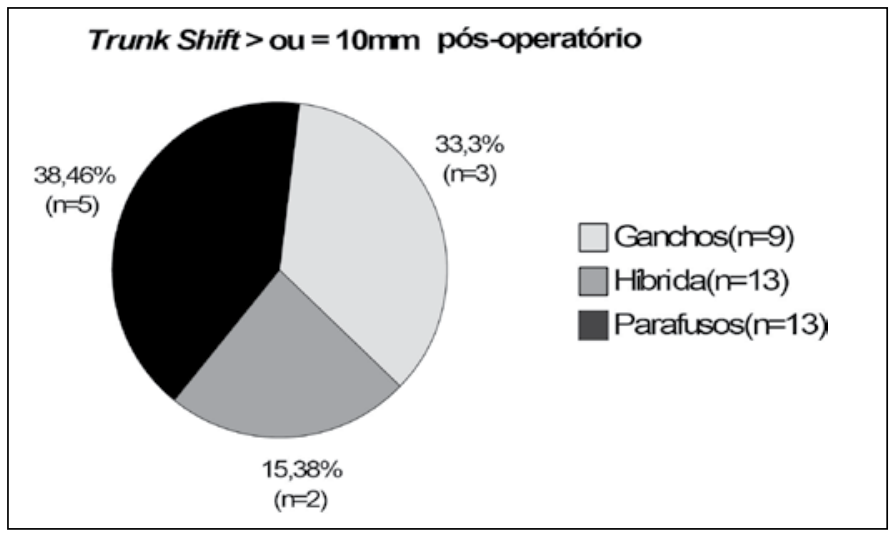

Fonte: Serviço de Arquivo Médico da ISCMSP - SAME.

Figura 4. Porcentagem das radiografias com trunk shift maior ou igual a $10 \mathrm{~mm}$ no pós-operatório.

\section{Descompensação Lateral do tronco no seguimento}

A descompensação maior ou igual a 10mm no seguimento mínimo de 1 ano observamos uma porcentagem próxima dos valores entre ganchos e parafusos (Figura 5)

A correção do tronco foi melhor com o uso da instrumentação de parafusos pediculares sendo observado desde o período do pós-operatório imediato para o seguimento mínimo de 1 ano, diminuindo a descompensação lateral esquerda para menor que $20 \mathrm{~mm}$ em todos os casos. Ocorreu a persistência da descompensação em alguns casos das instrumentações com ganchos e híbrida (Figura 6).

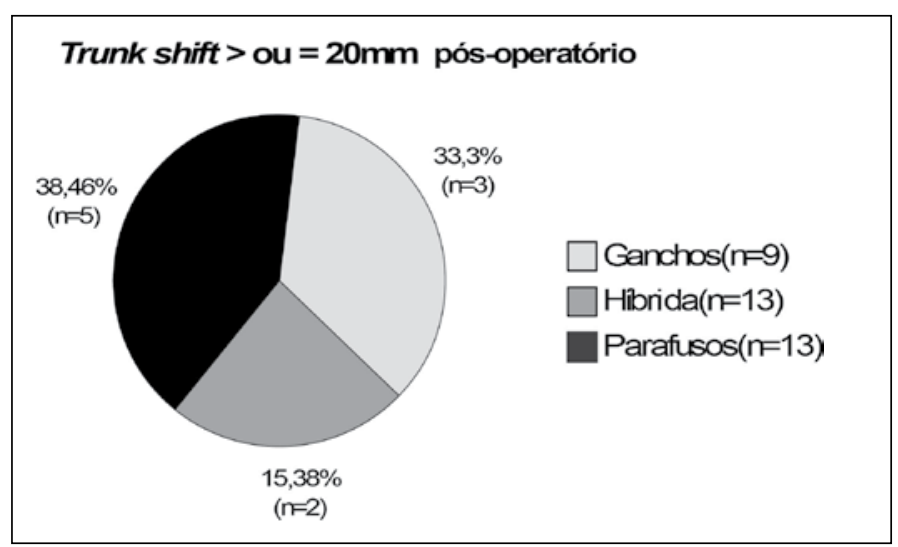

Fonte: Serviço de Arquivo Médico da ISCMSP - SAME.

Figura 5. Porcentagem das radiografias com trunk shift maior ou igual a $20 \mathrm{~mm}$ no pós-operatório.

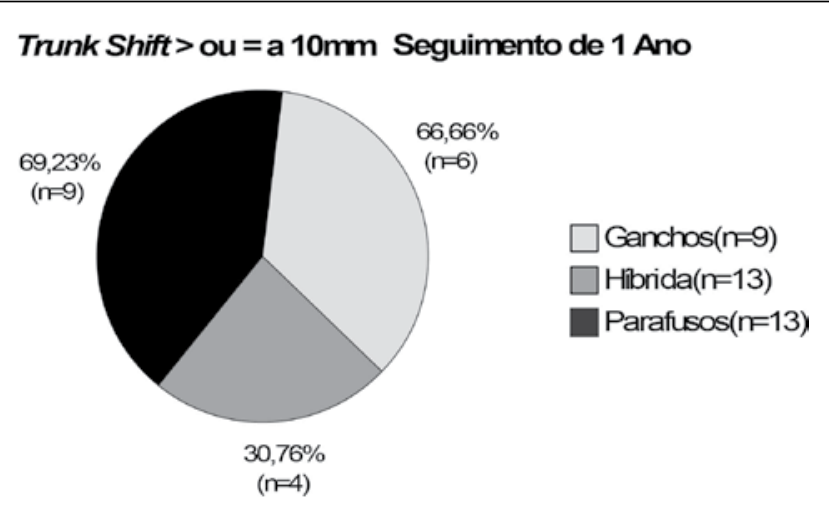

Fonte: Serviço de Arquivo Médico da ISCMSP - SAME.

Figura 6. Porcentagem das radiografias com trunk shift maior ou igual a $10 \mathrm{~mm}$ no seguimento de 1 ano. 
Em síntese, todos os casos com uso de somente parafusos pediculares apresentaram com trunk shift menor que $20 \mathrm{~mm}$. Verificamos desequilíbrio no grupo de ganchos em um paciente $(11,11 \%)$ e, na híbrida, em dois pacientes (15,38\%), entretanto não houve diferença estatisticamente significativa nestes achados Figura 7.

\section{Trunk shift $>$ ou $=20 \mathrm{~mm}$ Seguimento de $1 \mathrm{Ano}$}

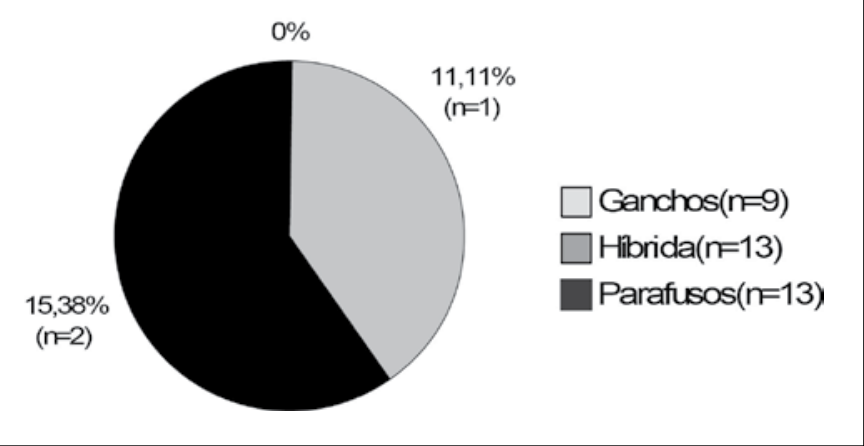

Fonte: SAME-ISCMSP.

Figura 7. Porcentagem das radiografias com trunk shift maior ou igual a 20 $\mathrm{mm}$ no seguimento de 1 ano.

\section{DISCUSSÃO}

A análise radiográfica comparativa do trunk shift entre as técnicas, com ganchos, híbrida e parafusos pediculares, dificilmente é encontrada na literatura. Nosso objetivo foi avaliar o desequilíbrio lateral do tronco sob este método nestes três grupos de instrumentação.

Richards et al. ${ }^{6}$ argumentaram que o deslocamento lateral do tronco é o real método de analisar este desequilíbrio coronal. A avaliação com esta técnica é observado em poucos estudos, sendo de preferência o uso da medida preconizada pela SRS para avaliação do equilíbrio do tronco.

A avaliação sob análise do equilíbrio coronal global preconizado pela SRS, pode ser observado em vários trabalhos comparativos. Lowenstein et al. ${ }^{9}$ em um estudo restrospectivo de 34 pacientes, demostraram nenhuma diferença estatística entre o uso de instrumentações entre parafusos e híbridas na descompensação do plano coronal. Dobbs et al. ${ }^{10}$ num estudo de 66 pacientes submetidos a artrodese seletiva torácica, após um seguimento de 2 anos foi observado uma melhor correção da descompensação para o uso com parafusos sendo uma média de $21 \mathrm{~mm}$ para ganchos e $17,3 \mathrm{~mm}$ para parafusos, $p<0,05^{10}$. Avanzi et al. ${ }^{11}$, em 22 pacientes submetidos a instrumentação de 11 hibrida e 11 ganchos, relataram uma média de descompensação pós-operatório imediato de 12,95mm e após o seguimento de 1 ano para 10,04mm. Na maioria dos trabaIhos foram observados poucas diferenças no desequilíbrio coronal entre o uso das variadas técnicas de instrumentação posterior sob a análise do equilíbrio coronal global.

$\mathrm{Na}$ análise do trunk shift, na nossa série, observamos nas radiografias o desequilíbrio do tronco da direita passando para esquerda após a cirurgia na grande maioria dos pacientes, como em outros estudos já realizados. A média no período pós-operatório imediato desta descompensação foi pouco diferente entre os três grupos sendo no período do pós-operatório imediato de $-16 \mathrm{~mm}$ ganchos, -12,8mm híbridas, -12,9mm parafusos, com uma correção no seguimento de 1 ano para $-9,9 \mathrm{~mm}$ para ganchos, $-11,8 \mathrm{~mm}$ híbrida e $-10,7 \mathrm{~mm}$ para parafusos. Richards et al. ${ }^{3,6}$, em seus primeiros estudos em 1992, com 24 pacientes submetidos a artrodese seletiva com uso de ganchos foram observados sob a análise do trunk shift o deslocamento para esquerda de $-15 \mathrm{~mm}$ no período pós-operatório imediato e a correção para -12mm após seguimento de 1 ano com uso de gancho. Luk et al. ${ }^{12}$ com pacientes submetidos a instrumentação de 25 parafusos e 25 híbridas obtiveram os resultados no período pós-operatório de $-7,9 \mathrm{~mm}$ para parafusos e $-11 \mathrm{~mm}$ para híbridas, e no seguimento $-8,3 \mathrm{~mm}$ para parafusos e $6 \mathrm{~mm}$ para híbridas. Em nosso trabalho, observamos que há uma mínima correção desta descompensação do tronco para esquerda do pós-operatório imediato para o segmento mínimo de um ano em todos os grupos.

No uso do instrumental com o uso de parafusos pediculares, todas as radiografias apresentaram com a medida do trunk shift menor que $20 \mathrm{~mm}$. Em poucos casos este desequilíbrio se apresentou, sendo no grupo de ganchos 1 paciente $(11,11 \%)$ e híbrida 2 pacientes (15,38\%). As instrumentações seletivas com somente parafusos pediculares mostraram raros casos de descompensação do tronco.

\section{CONCLUSÃO}

Houve ajuste do desequilíbrio do tronco após 1 ano da cirurgia na nossa série e que não houve diferença significativa no que concerne à descompensação lateral do tronco entre o uso das três técnicas de instrumentação.

\section{REFERÊNCIAS}

1. King HA, Moe JH, Bradford DS, Winter RB. The selection of fusion levels in thoracic idiopathic scoliosis. J Bone Joint Surg Am. 1983:65(9):1302-13.

2. Lenke LG, Edwards CC 2nd, Bridwell KH. The Lenke classification of adolescent idiopathic scoliosis: how it organizes curve patterns as a template to perform selective fusions of the spine. Spine (Phila Pa 1976). 2003:28(20):S199-207.

3. Frez $\mathrm{R}$, Cheng JC, Wong EM. Longitudinal changes in trunkal balance after selective fusion of King II curves in adolescent idiopathic scoliosis. Spine (Phila Pa 1976). 2000;25(11):1352-9

4. Richards BS. Lumbar curve response in type II idiopathic scoliosis after posterior instrumentation of the thoracic curve. Spine (Phila Pa 1976). 1992;17(8 Suppl):S282-6.

5. Li M, Shen Y, Fang X, Ni J, Gu S, Zhu X, Zhang Z. Coronal and sagittal plane correction in patients with Lenke 1 adolescent idiopathic scoliosis: a comparison of consecutive versus interval pedicle screw placement. J Spinal Disord Tech.2009;22(4):251-6.

6. Richards BS, Scaduto A, Vanderhave K, Browne R. Assessment of trunk balance in thoracic scoliosis. Spine (Phila Pa 1976). 2005;30(14):1621-6.

7. Bassett GS, Bunnell WP. Effect of a thoracolumbosacral orthosis on lateral trunk shift in idiopathic scoliosis. J Pediatr Orthop. 1986;6(2):182-5.
8. Newton PO, Faro FD, Lenke LG, Betz RR, Clements DH, Lowe TG, et al. Factors involved in the decision to perform a selective versus nonselective fusion of Lenke 1B and 1C (King-Moe II) curves in adolescent idiopathic scoliosis. Spine (Phila Pa 1976). 2003;28(20):S217-23.

9. Lowenstein JE, Matsumoto H, Vitale MG, Weidenbaum M, Gomez JA, Lee FY, et al. Coronal and sagittal plane correction in adolescent idiopathic scoliosis: a comparison between all pedicle screw versus hybrid thoracic hook lumbar screw constructs. Spine (Phila Pa 1976). 2007;32(4):448-52.

10. Dobbs MB, Lenke LG, Kim YJ, Kamath G, Peelle MW, Bridwell KH. Selective posterior thoracic fusions for adolescent idiopathic scoliosis: comparison of hooks versus pedicle screws. Spine (Phila Pa 1976). 2006;31(20):2400-4.

11. Avanzi O, Landim E, Meves R, Cafaro MFS, Umeta RSG, Kruppa, JTP. Avaliação radiográfica da descompensação do tronco após artrodese seletiva torácica em portadores de escoliose idiopática do adolescente King II (Lenke B e C). Coluna/Columna. 2009;8(4):376-80.

12. Luk KD, Don AS, Chong CS, Wong YW, Cheung KM. Selection of fusion levels in adolescent idiopathic scoliosis using fulcrum bending prediction: a prospective study. Spine (Phila Pa 1976). 2008;33(20):2192-8. 